\title{
Infección nosocomial y del sitio quirúrgico en un hospital de tercer nivel (2002-2005)
}

\author{
Ballestero Diego R, Rebollo Rodrigo H*, Gutiérrez Baños JL, Aguilera Tubet C, \\ Zubillaga Guerrero S, Martín García B.
}

Servicio de Urología. *Servicio de Medicina Preventiva. Hospital Universitario Marqués de Valdecilla. Santander.

Actas Urol Esp. 2006;30(9):905-912

\section{RESUMEN}

INFECCIÓN NOSOCOMIAL Y DEL SITIO QUIRÚRGICO EN UN HOSPITAL DE TERCER NIVEL (2002-2005)

Introducción: La medida de las tasas de infección nosocomial constituye un indicador de calidad asistencial, permitiendo adoptar medidas de prevención y control. Se ha desarrollado un plan de vigilancia de infección nosocomial en los hospitales, demostrando ser un método eficaz para disminuir su incidencia.

Objetivo: Conocer los indicadores y características de la infección nosocomial en general y de la infección del sitio quirúrgico en particular en un servicio de Urología de forma global y por procedimientos.

Material y métodos: Estudio prospectivo mediante el sistema de vigilancia epidemiológica entre 2002-2005 en 4.618 pacientes ingresados al menos 24 horas, practicándose un total de 3.096 intervenciones.

Resultados: Se observó una tasa global de infección nosocomial del 6,10\%, de infección urinaria del 3,42\% y de infección del sitio quirúrgico del 2,81\%. De esta última por procedimientos, la cistectomía se sitúa en el 22,8\%, seguido de la cirugía del riñón y uréter $(6,6 \%)$ y de la cirugía abierta de próstata $(4,36 \%)$.

Los gérmenes más frecuentemente aislados en la infección del sitio quirúrgico son Escherichia Coli (43,6\%) y Pseudomonas aeruginosa (15\%). En la infección urinaria los gérmenes más frecuentes son Escherichia Coli (43,6\%) y Pseudomonas aeruginosa (15\%)

Conclusión: Las tasas de infección nosocomial son inferiores a los valores estandares publicados. La mayor tasa de infección del sitio quirúrgico se presenta en las cirugías más complejas técnicamente, siendo Escherichia Coli, el agente etiológico más frecuente. La vigilancia de la infección del sitio quirúrgico y factores relacionados permiten incorporar elementos de mejora en la práctica clínico-quirúrgica, aportando un indicador de referencia en análisis posteriores.

Palabras clave: Control de infección. Infección de herida quirúrgica. Infección del tracto urinario.

\section{ABSTRACT}

NOSOCOMIAL INFECTION AND INFECTION OF THE SURGICAL SITE IN A THIRD LEVEL HOSPITAL (2002-2005)

Introduction: Nosocomial infection rates constitute an indicator of welfare quality, permitting to adopt measures of prevention and control. It has been developed a surveillance plan of the nosocomial infection in hospitals, showing to be an efficient method to diminish its incident.

Objective: To know the indicators and characteristics of the nosocomial infection and of the infection of the site surgical particularly, in a urology service in a global form and by procedures.

Matherial and methods: Prospective study by means of the epidemiological surveillance system from 2002 to 2005 in 4.618 patients hospitalised at least 24 hours, with a total of 3.096 surgical.

Results: The overall incidence of nosocomial infection was $6,10 \%, 3.42 \%$ for urinary infection and $2,81 \%$ for the infection of the chirurgical site. For procedures, the incidence of the infection of the surgical site for cistectomy was $22,8 \%$, $6,6 \%$ for surgery of kidney and ureter and $4,36 \%$ for open surgery of prostate.

Eschericia Coli $(43,6 \%)$ was the most frequently isolated organism, accounting for $43,6 \%$ of the causative organisms in the infection of the surgical site and $43,6 \%$ in the urinary infection. Pseudomonas aeruginosa is the next organism in frecuency with a $15 \%$ in both infections.

Conclusion: Our nosocomial infection rates are lower than the published standard values. The main infection rate of the surgical sites present in the most complex surgical techniques, whereas Escherichia Coli is the most frecuently isolated ethiological agent. The surveillance of the infection of the surgical site and related factors permit to incorporate improvements in the clinical-surgical practice which will be an indicator of reference in subsequent analysis. 
$\mathrm{L}$ a infección nosocomial (IN) y sobre todo la infección del sitio quirúrgico (ISQ) no han dejado de ser un problema para la Medicina desde que se tiene conocimiento histórico de las intervenciones quirúrgicas.

A pesar de las medidas de asepsia, de las mejoras en la técnica quirúrgica y de la profilaxis antibiótica; el aumento de edad de nuestros pacientes y su comorbilidad, el mayor número de técnicas de diagnóstico y tratamiento agresivas y el aumento de las resistencias microbianas hacen, entre otros factores, que las IN sigan constituyendo hoy en día uno de los mayores problemas en términos de morbimortalidad y coste económico al que se enfrentan las instituciones sanitarias.

En España, como en otros países de nuestro entorno, se calcula que entre un 6 y un 14\% de los pacientes que ingresan en los hospitales de agudos, desarrollan una $\mathrm{IN}^{1,2}$.

La infección del sitio quirúrgico influye de forma importante en el aumento de la estancia media hospitalaria, en las complicaciones y reintervenciones, en el bienestar del paciente y, en el aumento de los costes originados para el sistema nacional de salud ${ }^{3}$

Se calcula que la ISQ produce un alargamiento de la estancia entre 8 y 24 días ${ }^{1,4,5}$. LizánGarcía et al. en el servicio de Urología del Hospital General de Albacete, estiman 10 días extras de estancia por ISQ con un coste de 15.641 pts (94 euros)/día en $1998^{6}$.

Las tasas de ISQ, especialmente en cirugía limpia, se consideran un indicador de resultado fiable y permiten comparaciones internas a lo largo del tiempo y externas con otros hospitales. Es necesario monitorizar y cuantificar estas tasas mediante sistemas de vigilancia y control.

Estos sistemas son una estrategia efectiva del control de costes en el sistema sanitario y repercuten directamente en la mejora de la calidad asistencial que este sistema presta ${ }^{7}$. La implantación de un sistema de vigilancia dentro de un hospital constituye un plan multidisciplinar que integra servicios y trabajadores (desde la dirección del hospital, cirujanos, enfermeras y servicios encargados de la vigilancia).

No sólo la elevada frecuencia de estas infecciones sino la importancia con la morbimortali- dad y costes, así como la posibilidad real de ser prevenidas, justifican la realización de estudios de vigilancia y control de la ISQ. No existen muchos estudios publicados por cirujanos de tasas de IN o de ISQ en la bibliografía, y más concretamente en el campo de la urología ${ }^{8}$.

Nuestro objetivo es conocer la evolución de las tasas de IN, y su etiología en nuestro Servicio de Urología de forma global y por procedimientos, para detectar problemas y plantear las medidas oportunas de control, a partir de los datos obtenidos del sistema de vigilancia.

\section{MATERIAL Y MÉTODOS}

\section{Marco}

Nuestro ámbito de estudio es un servicio de urología de un hospital de tercer nivel con una media diaria de 30 camas. Se incluyeron en el estudio un total de 4.618 pacientes ingresados en el periodo, en el que se practicaron un total de 3.096 intervenciones quirúrgicas en estos pacientes ingresados.

\section{Método de vigilancia}

El periodo recogido en el estudio abarca desde el 1 enero del 2002 al 31 de diciembre del 2005.

La recogida de datos se realizó por parte del servicio de Medicina Preventiva del hospital mediante un sistema prospectivo de vigilancia epidemiológica de incidencia acumulada de IN global y por procedimientos quirúrgicos de los pacientes ingresados, operados o no, que pasan al menos 24 horas en el servicio de Urología. Las fuentes de información utilizadas son las historias clínicas, los volantes de microbiología y la visita diaria de miembros del servicio de medicina preventiva que tienen contacto directo con los responsables del paciente

Estos datos de incidencia acumulada se obtuvieron para el total de infecciones así como sus localizaciones más frecuentes (infección del sitio quirúrgico, urinaria, bacteriemia, etc)

El estudio de las localizaciones se hizo mediante una distribución porcentual referido al total de infecciones de cada año. Esto mismo se hizo con los microorganismos responsables de dichas infecciones.

Paralelamente se han recogido los datos de cumplimiento de profilaxis antibiótica, mante- 
niéndose intacta la política antibiótica en la profilaxis durante el estudio.

Se realizó de forma sistemática un urocultivo de control a todos los pacientes al ingreso.

Las definiciones de infección nosocomial se han basado en las recomendadas por el Center for Disease Control $(C D C)^{9,10}$.

Se incluyen los datos de incidencia acumulada obtenidos en estos cuatro años, así como los agentes etiológicos más frecuentes.

\section{RESULTADOS}

Del 1 de Enero del 2002 al 31 de Diciembre del 2005 se realizó el estudio prospectivo que incluyó un total de 4.618 pacientes ingresados al menos 24 horas en el Servicio de Urologia.

El número de intervenciones quirúrgicas en dicho periodo fue de 3.096 practicadas en estos pacientes ingresados.

Analizando de forma global (Tabla 1) obtenemos una tasa de IN global de $6,10(5,59-6,62)$ en 282 pacientes a lo largo del estudio, sin variaciones importantes en el periodo. Señalar que progresivamente se ha ido aumentado el número de ingresos y de pacientes intervenidos sin un aumento significativo en las tasas de IN.

Desglosando por tipos de IN (Tabla 2 y Figs. 1, 2 y 3), la tasa de infección urinaria se sitúa como

Tabla 1

Tasa de infección nosocomial expresados en \%

\begin{tabular}{lccccc}
\hline & $\mathbf{2 0 0 2}$ & $\mathbf{2 0 0 3}$ & $\mathbf{2 0 0 4}$ & $\mathbf{2 0 0 5}$ & Global \\
\hline № ingresos & 1178 & 1109 & 1126 & 1205 & 4618 \\
№ intervenciones & 712 & 740 & 799 & 845 & 3096 \\
№ IN & 78 & 64 & 63 & 77 & 282 \\
Tasa IN (\%) & $\mathbf{6 . 6 2}$ & $\mathbf{5 . 7 8}$ & $\mathbf{5 . 5 9}$ & $\mathbf{6 . 3 9}$ & $\mathbf{6 , 1 0}$ \\
\hline
\end{tabular}

IN: Infección nosocomial

Tabla 2

Tasa expresada en \% de incidencia localización de la IN

\begin{tabular}{lccccc}
\hline & $\begin{array}{l}\mathbf{2 0 0 2} \\
\text { \% (n) }\end{array}$ & $\begin{array}{l}\mathbf{2 0 0 3} \\
\mathbf{\%}(\mathbf{n})\end{array}$ & $\begin{array}{c}\mathbf{2 0 0 4} \\
\mathbf{\%}(\mathbf{n})\end{array}$ & $\begin{array}{l}\mathbf{2 0 0 5} \\
\mathbf{\%}(\mathbf{n})\end{array}$ & $\begin{array}{c}\text { Global } \\
\mathbf{\%}(\mathbf{n})\end{array}$ \\
\hline $\begin{array}{l}\text { Infección herida } \\
\text { quirúrgica }\end{array}$ & $2,25(16)$ & $2,97(22)$ & $2,75(22)$ & $3,20(27)$ & $2,81(87)$ \\
Infección urinaria & $4,07(48)$ & $3,25(36)$ & $2,75(31)$ & $3,57(43)$ & $3,42(158)$ \\
Infección respiratoria & $0,25(3)$ & $0,09(1)$ & $0,18(2)$ & $0,08(1)$ & $0,15(7)$ \\
Bacteriemia & $0,76(9)$ & $0,27(3)$ & $0,27(3)$ & $0,41(5)$ & $0,42(20)$ \\
Otros & $0,17(2)$ & $0,18(2)$ & $0,44(5)$ & $0,08(1)$ & $0,21(10)$ \\
\hline
\end{tabular}

IN: Infección nosocomial

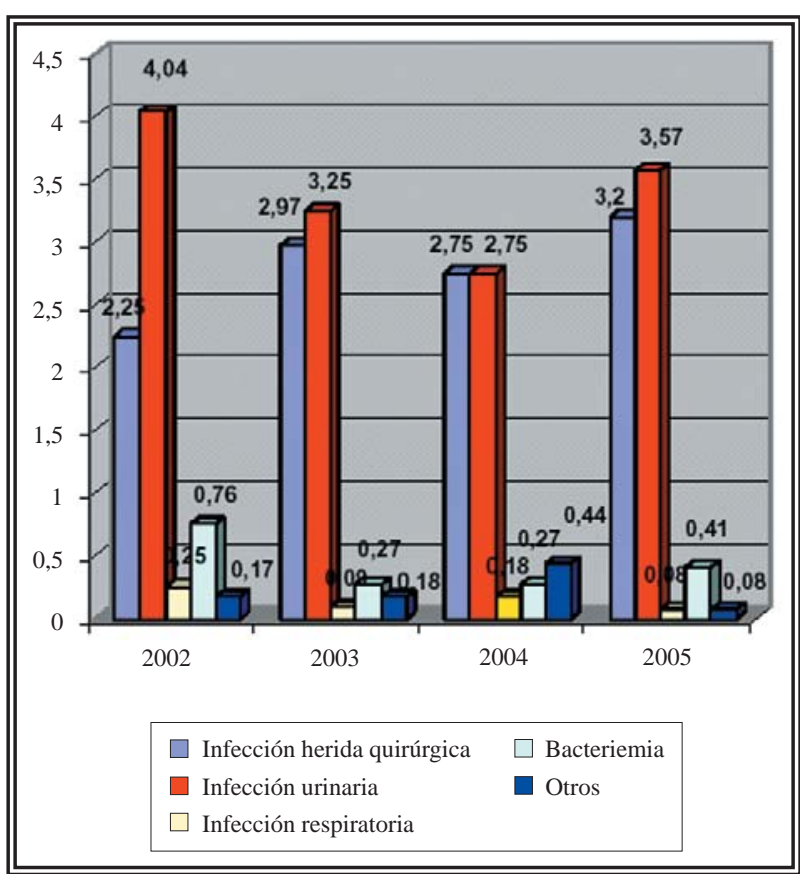

FIGURA 1. Tasa de IN por localización.

la más frecuente en 3,42 (2,75-4,07);la tasa de infección de herida quirúrgica se sitúa en 2,81 $(2,25-3,20)$, siendo el valor más alto en el año 2004. Obtenemos una tasa de infección respiratoria de $0,15(0,08-0,25)$, La tasa de bacteriemia es del $0,42(0,27-0,76)$ y la tasa de otras infecciones se sitúa en 0,21 $(0,44-0,08)$. Reseñar que en ninguno de los apartados anteriores existen variaciones interanuales significativas.

Analizando de una forma más concreta la infección de herida quirúrgica, desglosaremos la incidencia de la ISQ por el tipo de procedimiento quirúrgico (Tablas 3 y 4).

En cirugía del riñón y uréter obtenemos una tasa de ISQ de $6,68(5,34-8,72)$, observando una tendencia creciente a lo largo del estudio, aunque dentro de valores aceptables.

Con respecto a la cirugía endoscópica, únicamente destacar un único caso de ISQ debido a la perforación intraperitoneal de vejiga y posterior peritonitis en una resección transuretral de vejiga. 


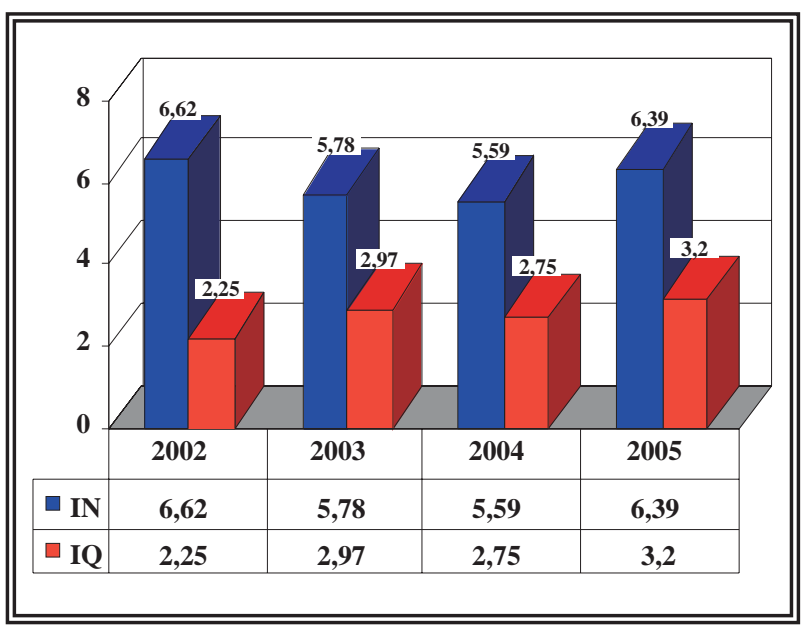

FIGURA 2. Sistema de vigilancia hospitalaria. Tasas glo bales.

En la cirugía abierta de próstata la tasa global se sitúa en 4,36 (2,06-7), destacando un valor anormalmente alto en el año 2003 en relación a un insuficiente cumplimiento en la profilaxis antibiótica, sobre todo en pacientes sondados.

En el apartado de cistectomías y enterocistoplastias la tasa de IQ se sitúa en 22,86 (15,7935).

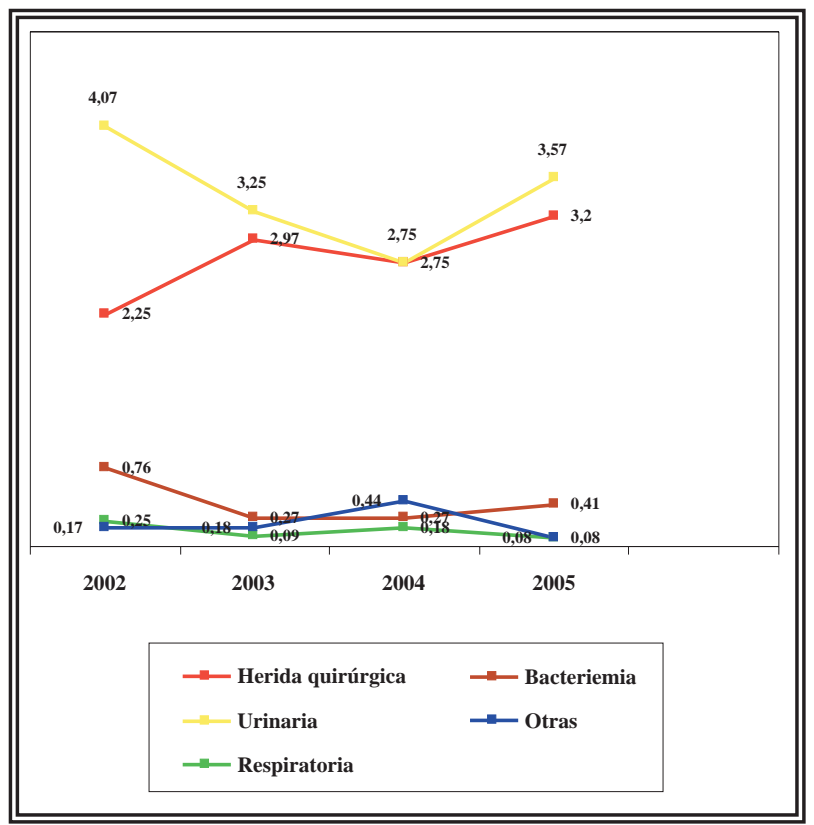

FIGURA 3. Evolución de tasas de infección nosocomial por localización.

En cirugia genital la tasa media es de $1,53(0,91$ 2,21 ), recordando que en el estudio sólo se incluyen los pacientes ingresados al menos 24 horas, lo que excluye a todos los pacientes ambulatorios.

Tabla 3

Gérmenes aislados en la ISQ por procedimientos

\begin{tabular}{|c|c|c|c|c|}
\hline $\begin{array}{l}\text { Cirugia por } \\
\text { procedimientos }\end{array}$ & $\begin{array}{l}2002 \\
\text { Tasa ISQ } \\
\text { Gérmen más } \\
\text { frecuente }\end{array}$ & $\begin{array}{l}2003 \\
\text { Tasa ISQ } \\
\text { Gérmen más } \\
\text { frecuente }\end{array}$ & $\begin{array}{l}2004 \\
\text { Tasa ISQ } \\
\text { Gérmen más } \\
\text { frecuente }\end{array}$ & $\begin{array}{l}2005 \\
\text { Tasa ISQ } \\
\text { Gérmen más } \\
\text { frecuente }\end{array}$ \\
\hline Riñón y uréter & $\begin{array}{l}5,34 \\
\text { S aureus }\end{array}$ & $\begin{array}{l}6,77 \\
\text { E.coli/Pseudomona } \\
\text { aeurginosa }\end{array}$ & $\begin{array}{l}6,29 \\
\text { E. coli/Enterococus } \\
\text { faecalis }\end{array}$ & $\begin{array}{l}8,72 \\
\text { E. coli/Enterococus } \\
\text { faecalis }\end{array}$ \\
\hline RTU (vejiga y próstata) & $\begin{array}{l}0,38^{*} \\
\text { E. coli/Ps. aeruginosa }\end{array}$ & 0,00 & 0,00 & 0,00 \\
\hline Cirugía abierta de próstata & $\begin{array}{l}2,80 \\
\text { Enterococus vaginalis }\end{array}$ & $\begin{array}{l}7,00 \\
\text { Ps. Aeruginosa/ }\end{array}$ & $\begin{array}{l}2,06 \\
\text { E. Coli } \\
\text { Enterococus faecalis }\end{array}$ & $\begin{array}{l}5,50 \\
\text { Ps. Aeruginosa/ E. Coli }\end{array}$ \\
\hline Cistectomía/Enterocistoplastia & $\begin{array}{l}16,67 \\
\text { E. coli }\end{array}$ & $\begin{array}{l}23,08 \\
\text { E. coli }\end{array}$ & $\begin{array}{l}35,00 \\
\text { E. coli }\end{array}$ & $\begin{array}{l}15,79 \\
\text { E. coli }\end{array}$ \\
\hline Cirugia genital & $\begin{array}{l}1,98 \\
\text { Ps. aeruginosa }\end{array}$ & $\begin{array}{l}2,21 \\
\text { Acinetobacter } \\
\text { baumanii E coli/Ps. } \\
\text { Aeruginosa/ }\end{array}$ & $\begin{array}{l}1,01 \\
\text { E. coli }\end{array}$ & $\begin{array}{l}0,91 \\
\text { Flora } \\
\text { bacteriana } \\
\text { mixta. }\end{array}$ \\
\hline Otros & 0,00 & 0,00 & $\begin{array}{l}0,97 \\
\text { Pseudomona } \\
\text { Aeruginosa }\end{array}$ & $\begin{array}{l}2,02 \\
\text { Staphylococcus } \\
\text { epidermidis }\end{array}$ \\
\hline
\end{tabular}

* 1 Peritonitis

ISQ: Infección del sitio quirúrgico 
Tabla 4

Gérmenes más frecuentemente aislados

\begin{tabular}{lclc}
\hline $\begin{array}{l}\text { Infección del sitio quirúrgico } \\
\text { Germen }\end{array}$ & $\%$ & Germen & Infección urinaria \\
\hline Escherichia Coli & 43,6 & Escherichia Coli & 45,6 \\
Pseudomonas Aeruginosa & 15 & Pseudomonas Aeruginosa & 10,1 \\
Enterococcus Faecalis & 12,6 & Enterococcus Faecalis & 9,5 \\
Staphylococcus Aureus & 11,4 & Staphylococcus Epidermidis & 3,8 \\
Staphylococcus Epidermidis & 4,6 & Proteus Mirabilis & 3,8 \\
\hline
\end{tabular}

Finalmente en el apartado otros se incluyen técnicas como los TVT, TOT, biopsias de próstata y esfinteres artificiales. La tasa de IQ en este grupo es de 0,75/0,84 (0-2,02)

Analizando los agentes etiológicos de la infección quirúrgica por procedimientos observamos (Tablas 3 y 4) que en la cirugia del riñón y del uréter los gérmenes más frecuentemente aislados son las bacterias gram negativas, sobre todo Escherichia Coli y Enterococcus faecalis. En la cirugia endoscópica, cirugía abierta de próstata, cirugía genital y en el apartado de cistectomías Escherichia Coli representa el germen más frecuentemente aislado, siendo Pseudomona Aeruginosa el segundo germen en frecuencia.

Globalmente los gérmenes más frecuentemente aislados son (Tabla 5) Eschericia Coli $(43,6 \%)$, Pseudomonas aeruginosa (15\%), Enterococcus faecalis $(12,6 \%)$, Staphylococcus Aureus $(11,4 \%)$ y Staphylococcus Epidermidis $(4,6 \%)$.

Con respecto a la infección urinaria (Tabla 5), las bacterias gram negativas son los gérmenes aislados con mayor frecuencia, siendo Escherichia Coli el más frecuente $(45,6 \%)$, seguido por Pseudomona aeruginosa $(10,1 \%)$, Enterococcus faecalis $(9,5 \%)$, Staphylococcus epidermidis y Proteus mirabilis $(3,8 \%)$, coincidentes con los publicados en la bibliografia.

Tabla 5

Gérmenes aislados en la infección urinaria por procedimientos

\begin{tabular}{|c|c|c|c|c|}
\hline $\begin{array}{l}\text { Cirugia por } \\
\text { procedimientos }\end{array}$ & $\begin{array}{c}2002 \\
\text { Tasa IU } \\
\% \text { (n/total) } \\
\text { Gérmen más } \\
\text { frecuente }\end{array}$ & $\begin{array}{c}2003 \\
\text { Tasa IU } \\
\text { \% (n/total) } \\
\text { Gérmen más } \\
\text { frecuente }\end{array}$ & $\begin{array}{c}2004 \\
\text { Tasa IU } \\
\% \text { (n/total) } \\
\text { Gérmen más } \\
\text { frecuente }\end{array}$ & $\begin{array}{c}2005 \\
\text { Tasa IU } \\
\% \text { (n/total) } \\
\text { Gérmen más } \\
\text { frecuente }\end{array}$ \\
\hline Riñón y uréter & $\begin{array}{l}4,58(6 / 131) \\
\text { E.Coli }\end{array}$ & $\begin{array}{c}8,27(11 / 133) \\
\text { E.Coli/ Ps aeruginosa }\end{array}$ & $\begin{array}{c}5,71(10 / 175) \\
\text { E. coli/ Ps aeruginosa }\end{array}$ & $\begin{array}{c}\text { 4,65 }(8 / 172) \\
\text { E. coli/ } \\
\text { Ps Aeruginosa }\end{array}$ \\
\hline RTU (vejiga y próstata) & $\begin{array}{l}8,68(23 / 265) \\
\text { E.coli }\end{array}$ & $\begin{array}{l}3,99(11 / 276) \\
\text { E.Coli }\end{array}$ & $\begin{array}{c}3,61(11 / 305) \\
\text { E.Coli/ E. Cloacae }\end{array}$ & $\begin{array}{c}\text { 3,87 }(13 / 336) \\
\text { E. Coli, } \\
\text { E. Faecalis }\end{array}$ \\
\hline Cirugía abierta de próstata & $\begin{array}{c}6,54(7 / 107) \\
\text { E.Coli/ Ps. Aeruginosa }\end{array}$ & $\begin{array}{l}\quad 6,00(6 / 100) \\
\text { E. faecalis / S. epidermidis }\end{array}$ & $\begin{array}{c}4,12(4 / 97) \\
\text { E.Coli/ S.epidermidis }\end{array}$ & $\begin{array}{l}15,60(17 / 109) \\
\text { E. coli }\end{array}$ \\
\hline Cistectomia/Enterocistoplastia & $\begin{array}{l}\text { 16,67 }(3 / 18) \\
\text { E. coli }\end{array}$ & $\begin{array}{c}7,69(1 / 13) \\
\text { Hongos levaduriformes }\end{array}$ & $\begin{array}{l}10,00(2 / 20) \\
\text { E. coli }\end{array}$ & $0(0 / 3)$ \\
\hline Cirugía genital & $0(0 / 101)$ & $\begin{array}{c}1,47(2 / 136) \\
\text { E.coli/ S. epidermidis }\end{array}$ & $0(0 / 99)$ & $\begin{array}{c}0,91 \\
\text { Ps Aeruginosa }\end{array}$ \\
\hline Otros $0(0 / 90)$ & $2,44(2 / 82)$ & $\begin{array}{c}2,91(3 / 103) \\
\text { E.Coli/ Ps aeruginosa }\end{array}$ & $\begin{array}{c}1,01 \\
\text { Ps. Aeruginosa/ } \\
\text { P. Mirabilis/ A. Baumanii }\end{array}$ & K. Pneumoniae \\
\hline
\end{tabular}

IU: Infección urinaria.

E. Coli: Escherichia Coli

Ps: Pseudomona.

E. Faecalis: Enterococo faecalis

K. Pneumoniae: Klebsiella Pneumoniae

A. Baumanii: Acinetobacter Baumanii

S. Epidermidis: Estafilococo Epidermidis

E. Cloacae: Enterobacter Cloacae 


\section{DISCUSIÓN}

La vigilancia de la infección nosocomial sirve para evaluar la calidad de la atención sanitaria. Hasta la fecha se ha comprobado en todos los estudios que la recogida de datos y su análisis sirven para reducir dichas tasas, consiguiendo de esta manera disminuir las complicaciones del paciente, la estancia hospitalaria y los gastos concomitantes. Estas infecciones pueden reducirse con programas de vigilancia y control efectivos que incluyan la comunicación de las tasas a los cirujanos y al personal de enfermería ${ }^{11}$.

Estos programas deben ser un objetivo multidisciplinar que afecte a la dirección del hospital, a los cirujanos, personal de enfermería y personal encargado de la vigilancia, que será el que controle y calcule las tasas de infección a lo largo del tiempo, detecte problemas puntuales y proponga medidas de mejora ${ }^{12}$.

Creemos que este trabajo resalta la eficacia de los sistemas de vigilancia para disminuir el riesgo de la IN, siendo un arma muy útil para la educación continuada del personal que trata a los pacientes.

Las tasas de infección quirúrgica cumplen las características necesarias de todo indicador: son un suceso clave en el ámbito hospitalario; tienen criterios de definición bien establecidos, utilizando como referencia los estipulados por el CDC de Atlanta (Center for Disease Control and Prevention) ${ }^{9,10}$; la puesta en práctica se llevaría a cabo a través de los programas de control de la infección mediante la búsqueda activa y directa de los pacientes intervenidos, y la utilidad queda patente al reflejar la situación de la asistencia sanitaria y establecer donde se debe incidir en las medidas de prevención de la infección, en contraste con los factores de riesgo del paciente, intrínsecos, y por lo tanto poco modificables.

Destacar en nuestro análisis la dificultad en la comparación entre hospitales y estudios, debido a las diferencias existentes entre los sistemas de vigilancia llevados a cabo por cada uno de ello. Incluso dentro del mismo hospital, el riesgo de adquirir una IN presenta variaciones importantes según el servicio en el que ingrese, siendo en general mayores las tasas de los servicios quirúrgicos que la de los médicos ${ }^{13}$.

En nuestro estudio se observa una disminución en la incidencia global de IN, especialmente de infecciones urinarias.
Coincidimos con otros estudios en las localizaciones más frecuentes de la IN, que por orden de frecuencia son: La infección urinaria, la ISQ y la bacteriemia ${ }^{10,14}$.

Las tasas de incidencia de IN y ISQ son inferiores a los valores estándares publicados incluyendo el grupo de cistectomías y enterocistoplastias que presenta tasas de ISQ con tendencia creciente en los años analizados, pero por debajo de otros valores comparados.

La mayor tasa de ISQ corresponde, como era previsible, a las cirugías más complejas técnicamente (riñón, uréter y cistectomías-enterocistoplastias).

Con respecto a las tasas de ISQ en cistectomías, Takeyama et al. informan de una tasa de incidencia del 33\% siendo el Staphylococcus aureus el germen aislado con más frecuencia ${ }^{15}$. Por el contrario Yamamoto presenta una tasa de $23,30 \%$. Sin embargo no podemos establecer comparaciones por no describirse en función de la técnica quirúrgica y derivación urinaria empleada ${ }^{16}$.

Existen pocos estudios que aporten datos comparativos con respecto a la infección urinaria por procedimiento quirúrgico. Con respecto a estas tasas en las RTU de vejiga y próstata, Pestalozzi et al. aportan unas tasas de $3 \%$ y del $2-5 \%$ respectivamente y un $57 \%$ en pacientes sondados ${ }^{17}$. Rassweiler et al. en su revisión del año 2006 informan de unas tasas entre el 1,7 y el $8,2 \%$, todas ellas en el rango de las cifras de nuestro estudio ${ }^{18}$. Señalar que no hemos establecido diferencias con respecto a diferentes variables tales como el sondaje y la bacteriuria previa, factores que aumentan el riesgo de ITU ${ }^{17,18}$.

En el grupo de cirugía del riñón y uréter, las tasas publicadas oscilan entre el 1,04 que establece el índice de NNIS y el 8,90 de Yamamoto et al. en nefroureterectomías ${ }^{10,16}$. El grupo Epine 2003 obtiene unos valores de $5,45 \%{ }^{19}$. Señalar en este apartado que no se ha diferenciado en este grupo entre cirugía laparoscópica y abierta, por ser reciente el inicio de la cirugía laparoscópica en nuestro servicio. Con respecto a este último grupo Brook NR presenta unas tasas de ISQ de 2,86 mientras que la tasa del grupo de Yamamoto et al. se sitúa en $1,40 \%$ en nefrectomías laparoscópicas ${ }^{16,20}$. 
Con respecto a la cirugía abierta de próstata, Epine 2003 presenta un valor de referencia de $5,33 \%$, similar a Yamamoto et al. que presenta un $6 \%$ para la prostatectomía radical ${ }^{16,19}$. El indice de NNIS establece este valor en 1,3910.

Las infecciones urinarias son las IN más frecuentes, representando junto a las ISQ y las bacteriemias más del 90\% de las mismas, que coincide con estudios previos.

La incidencia publicada de infecciones urinarias en pacientes urológicos varía entre el 1 y el $20 \%$. Pocos datos se han publicado al respecto específicamente en pacientes urológicos. Wenzel et al. revelaron un $6,2 \%^{21}$. Westenfelder et al. reportaron una incidencia de $21,4 \%$, mientras que Merle et al. observaron un 6,9\% ${ }^{12,22}$. Nuestra frecuencia se halla en un 3,42\%, aunque existe un problema de extrapolación de las muestras al no conocer el porcentaje de pacientes sondados, variable que aumenta esta tasa de infección.

Respecto a los germenes productores de infección del ISQ y urinaria coinciden con la bibliografía, excepto en la aparición de Acinetobacter baumanii en 2003, en relación a un brote en la UCI del hospital. Monge $\mathrm{V}$ et al. difieren únicamente en un alto porcentaje de infección urinaria por Serratia marcescens ${ }^{13}$. Merle et al. en sus estudios aíslan como gérmenes más frecuentes $\mathrm{P}$. Aeruginosa y enterococcus, situándose E. Coli en tercer lugar con un 13,4\%11,12,14,23.

La información periódica a los servicios quirúrgicos de las tasas de IN y especialmente de ISQ específicas por procedimientos, permiten incorporar elementos de mejora en la práctica asistencial, aportando además, un indicador de resultado para los contratos de gestión y la evaluación de la calidad asistencial, así como niveles de referencia para análisis posteriores.

Sin embargo muchas preguntas siguen sin respuesta debido a la evidencia científica insuficiente y a la dificultad de estudios rigurosos.

\section{REFERENCIAS}

1. Haley RW, Schaberg DR, Crossley KB, von Allmen SD and McGowan JE Extra charges and prolongation of stay attributable to nosocomial infections: A prospective interhospital comparison. Am J of Med. 1981;70:51-58.

2. Moris de la Tassa J, Fernández Muñoz P, Antuña Egocheaga A, Gutiérrez del Río MC, Cartón Sánchez JA. Estimación de los costes asociados con la infección del tracto urinario. Un estudio de casos y controles. Rev Clin Esp 2003;203(3):119-124.
3. Monge Jodra V. Infección de la herida quirúrgica. Indicadores de calidad. Asepsia y Esterilización. Septiembre 2003; 46:10-17.

4. Coello R, Glenister H, Fererest J, Bartlett and Cooke EM. The cost of infection in surgical patients: a case-control study. J Hosp Infect. 1993;25(4):239-250.

5. Asensio Vegas A, Monge Jodrá V, Lizán García M. Nosocomial infection in surgical wards: a controlled study of increased duration of hospital stays and direct cost of hospitalization. Europ J Epidemiol. 1993;5:504-510.

6. Lizán-García M, Gallego C, Martínez I. La infección de localización quirúrgica: una aproximación al coste atribuible. Medicina Preventiva 2004,vol X:6-11.

7. Mangram AJ, Horan TC, Pearson ML, Silver LC, Jarvis WR. Guideline for Prevention of Surgical Site Infection 1999. Hospital Infection Control Practices Advisory Committee. Infect Control Hosp Epidemiol. 1999;20(4):250-278.

8. Monge Jodra V. Infección quirúrgica como indicador de calidad: Perspectiva epidemiológica. Ponencia en I Jornadas Internacionales y IV nacional sobre avances en Medicina Preventiva. Marzo 2001:43-47.

9. Centers for disease control and Prevention. National nosocomial Infection surveillance (NNIS) report, data summary from October 1986 - April 1996. Issued May 1996. Am J Infect Control 1996;24:380-388.

10. National Nosocomial Infections Surveillance (NNIS) System Report, data summary from January 1992 through June 2004, issued October 2004. Am J Infect Control. 2004;32 (8):470-485.

11. Aldana Espinal JM, Valencia R, Gasch A, Conde M, Flores JM, Murillo F. La infección nosocomial como indicador de calidad asistencial. Comunicación en el XII Congreso de la Sociedad Española de Medicina Preventiva, Salud Pública e Higiene. 210: 478.

12. Merle V, Germain J-M, Bugel H, Nouvellon M, Lemeland JF, Czernichow P et al. Nosocomial Urinary Tract Infections in Urologic Patients: Assessment of a Prospective Surveillance Program Including 10000 patients. European Urology 2002;41:483-489.

13. Monge Jodra V, Sanz Cuesta MT, Olalla García MT, Muñoz Carpio MF. Infección nosocomial en un servicio de Urologia: Tendencias temporales de la incidencia acumulada durante el periodo 1984-1988. Rev Clin Esp. 1991;89: 309-313.

14. Knopf HJ, Weib P, Schafer W, Funke PF. Nosocomial infection after transurethral prostatectomy. Eur Urol 1999;36; 207-212.

15. Takeyama K, Matsukawa M, Kunishima Y, Takahashi S, Hotta $\mathrm{H}$, Nishiyama $\mathrm{N}$ et al. Incidence of and risk factors for surgical site infection in patients with radical cystectomy with urinary diversion. $J$ Infect Chemother 2005;11(4): 177-181.

16. Yamamoto S, Kunishima Y, Kanamaru S, Ito N, Kinoshita $\mathrm{H}$, Kamoto $\mathrm{T}$ et al. A multi-center prospective study for antibiotic prophylaxis to prevent perioperative infections in urologic surgery. Hinyokika Kiyo. 2004;50(10):673-683.

17. Pestalozzi DM, Boss HP, Knonagel H. Infectious complications after transurethral resection. Helv Chir Acta 1992; 59(3):497-500.

18. Rassweiler J, Teber D, Kuntz R, Hofmann R. Complications of transurethral Resection of the prostate (TURP). Incidence, Management and Prevention. Eur Urol avalaible online 2006 Jan 30. Article in press. 
19. Brook NR, Harper SJ, Waller JR, Nicholson ML. A consecutive series of 70 laparoscopic donor nephrectomies demostrates the safety of this new operation. Transplant Proc. 2005;37(2):627-628.

20. Vaqué J. Grupo de trabajo EPINE 2004. Evolución de la prevalencia de las infecciones nosocomiales en los hospitales españoles: 177 y 200.

21. Wenzel RP, Osterman CA, Hunting KJ. Hospital-acquired infections. Part II. Infection rates by site, service and common procedures in a university hospital. Am J Epidemiol. 1976;104(6):645-651.

22. Westenfelder M, Rosset K, Pelz K. Development of nosocomial and iatrogenic urinary tract infections (UTI) following urological interventions. A prospective clinical study. Scand J Urol Nephrol Suppl. 1987;104:59-63.
23. Colau A, Lucet JC, Rufat P, Botto H, Benoit G, Jardin A. Incidence and risk factors of bacteriuria after transurethral resection of the prostate. Eur Urol. 2001;39:272276.

Dr. R. Ballestero Diego

Hospital Marqués de Valdecilla

Servicio de Urología

Avenida Marqués de Valdecilla, s/n

39008 Santander

E-mail: badiro2@yahoo.es

(Trabajo recibido el 26 de junio de 2006) 